\title{
Aplicaciones de la Teoría de la Actividad de Leontiev y Vygostki al Ámbito de las Competencias Docentes de los Profesores de Ética USACH
}

\section{Applications of the Activity Theory by Leontiev and Vygotski to the field of Educational Competencies of USACH professors of Ethics}

\author{
Patricia Isabel Villouta Osorio \\ Universidad de Santiago, Chile
}

(Rec.: mayo de 2016 - Acept.: marzo de 2017)

Resumen

El presente estudio indaga la actividad docente para el curso de Ética en la Facultad Tecnológica de la Universidad de Santiago de Chile. Su objeto es identificar a partir de aportes teóricos del Constructivismo, como Leplat, Vygotski y La Teoría de la Actividad de Leóntiev, el trabajo que realizan los profesores para desarrollar la asignatura. Se apunta al reconocimiento de fortalezas y debilidades presentes, en función de su horizonte formativo o contexto en el cual despliegan su quehacer. Este estudio se perfila basándose en el análisis cualitativo a través de la aplicación de diez entrevistas y dos grupos focales, efectuados a todos los Profesores con 3 años de experiencia en su ejercicio de Pregrado. A partir de esta teoría se comprendió la dinámica de la actividad docente, y a partir de ahí contemplarla dimensionalmente, de modo de ordenar, jerarquizar y detectar elementos que sirvan para proponer competencias docentes. Al mismo tiempo se detectaron ciertas falencias de la actividad, inserta dentro del contexto de la enseñanza en Ética.

Palabras clave: Vygotski, teoría de la actividad, Leóntiev, Asignatura de Ética, competencias.

\begin{abstract}
The present study investigates the teaching activity for the Ethics course at the Technological Faculty of the University of Santiago, Chile. Its purpose is to identify, from theoretical contributions of Constructivism, such as Leplat, Vygotski and The Theory of Activity of Leontiev, the work that teachers perform to develop the subject. It aims at the recognition of strengths and weaknesses present in function of their formative horizon or context in which they unfold their work. This study is based on the qualitative analysis, through the application of ten interviews and two focus groups, made to all Professors with 3 years of experience in their undergraduate program. From this theory the dynamics of the teaching activity was understood, and from there to contemplate it dimensionally so as to order, hierarchize and detect elements that serve to propose teaching competences. At the same time certain failures of the activity were detected, inserted within the context of the teaching in Ethics.
\end{abstract}

Keywords: Vygotski, activity theory, Leontiev, ethics, educational competencies.

\footnotetext{
* Correspondencia a: Patricia Villouta Osorio. Universidad de Santiago de Chile. Av. Ecuador 3726 Santiago de Chile.
} E-mail: patricia.villouta@usach.cl 


\section{Introducción}

Generalmente se habla de las competencias como una combinación dinámica de atributos, en relación a conocimientos, habilidades, actitudes y responsabilidades que describen los resultados de los aprendizajes de un programa educativo, o lo que los estudiantes son capaces de demostrar al final del proceso educativo . Las competencias tienden a transmitir el significado de lo que la persona es capaz de hacer o es competente para ejecutar, el grado de preparación, suficiencia o responsabilidad para ciertas tareas (Prieto, 2002, citado en Cano, 2008). No obstante, cabe preguntarse si estos aspectos son considerados a la hora de intervenir en las competencias de los docentes y, a consecuencia de ello, en los aprendizajes de los alumnos. Ante este escenario, es de interés entender y comprender dicho fenómeno dentro de la asignatura de Ética, impartida a tecnólogos de la Facultad Tecnológica (FACTEC) de la Universidad de Santiago.

La Facultad Tecnológica es una de las 8 facultades de la Universidad de Santiago de Chile, contando en total con 14 carreras y en cuya malla curricular. Hasta el año 2016, se impartía la asignatura de Ética para la carrera de Tecnólogo de la FACTEC. Dicha asignatura no está exenta, junto a otras, del impacto que el aprendizaje efectivamente pueda tener en los alumnos/as.

Este trabajo pretende ser un aporte teórico respecto a la forma en cómo se realiza la actividad docente y una ayuda no sólo para quienes enseñan en la asignatura, sino para los alumnos/ as, universidades y organizaciones que reciben a los egresados universitarios. Para ello se aplica la Teoría Histórico Cultural de Vygotski (1934), con objeto de entender la naturaleza de los signos y las herramientas que se vinculan dentro de este contexto formativo. Con posterioridad, se profundiza en la Teoría de la Actividad (TA) continuada por Leontiev (1983), quien emprende el reconocimiento de las modalidades operacionales a través de acciones y procedimientos, conforme al logro de objetivos. Eso permite ilustrar un mapa contextual de relaciones, pudiendo visibilizar desde este encuadre las debilidades que plantean nuevas formas de acción, y junto con ello una nueva realidad ante las competencias docentes, presentes y ausentes en ellos.

Remitiéndonos a los planteamientos elaborados por Vygotski (1934), hallamos que es a través de las relaciones sociales y el diálogo cooperativo donde se adquieren las competencias únicas culturalmente adaptativas, mediante las cuales se van formando estructuras psicológicas que se usarán para la conducta. No obstante, existe un rango de tareas que los niños aún no pueden dominar solos, pero que pueden lograr con la ayuda de adultos; a este concepto se le denominó Zona de Desarrollo Próximo (ZDP, Berck, 2006). 2

\footnotetext{
1 Proyecto Tuning: El proyecto Alfa Tuning América Latina busca "afinar" las estructuras educativas de América Latina iniciando un debate cuya meta es identificar e intercambiar información y mejorar la colaboración entre las instituciones de educación superior para el

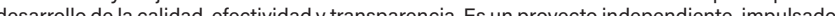
y coordinado por universidades de distintos países, tanto latinoamericanas como europeas.

2 Según Vygotski (1934), desde los 12-36 meses (Etapa Sensoriomotor) su contexto varía comenzando la actividad simbólica, primando la construcción del objeto, entre los 2-6 años (Etapa Personalismo), comienza a formar la imagen de sí mismo, entre los 6-11 años (Etapa (Etapa Personalismo), comienza a formar la imagen de sí mismo, entre los 6-11 años (Etapa más significativa y la Adolescencia, está en la construcción de su propio yo independiente.
}

Por otra parte, cabe distinguir que es por medio del juego simbólico donde los niños comienzan a darse cuenta que el pensar o el significado de las palabras, está separado de las acciones y objetos a los que remplaza, y las ideas pueden ser utilizadas para guiar las conductas (Vygotski, 1934).

Tomando en cuenta lo anterior, podemos comprender que los profesores, en su gran mayoría, son profesionales que no han cursado la carrera de Ética propiamente tal, por lo que han adquirido el conocimiento para enseñar dicha asignatura basándose en su propia experiencia profesional, o en cursos de perfeccionamiento. Por ello, al aplicar los planteamientos de Vygotski, relevamos que es a través de un nivel de desarrollo actual hasta uno próximo donde se van desarrollando sus competencias para la enseñanza en esta asignatura, en función de un desarrollo potencial.

En el estudio se observa un desplazamiento continuo que une el pensamiento de los docentes (para lograr su meta), con necesidades y comportamientos, juzgando las construcciones representacionales operatorias que definen, a modo personal, como adecuadas. Todo esto tiene lugar dentro de un proceso de ensayo y error que, como menciona Ochanine (1981), correspondería a niveles jerarquizados de apropiación de la realidad.

2 Según Vygotski (1934), desde los 12-36 meses (Etapa Sensoriomotor) su contexto varía comenzando la actividad simbólica, primando la construcción del objeto, entre los 2-6 años (Etapa Personalismo), comienza a formar la imagen de sí mismo, entre los 6-11 años (Etapa Categorial), el pensamiento del niño está más organizado en un conocimiento de la realidad más significativa y la Adolescencia, está en la construcción de su propio yo independiente.

Complementariamente, Leontiev (1983) nos señala que la actividad es un fenómeno: propositivo, contextual, contingente, constructivo, transformador, multinivel, jerarquizado y plástico (Díaz, 2008). Para efectos de este estudio, se trabajó con la actividad en función de satisfacer las necesidades por medio del logro de objetivos y metas. En consecuencia, se aplicó el fenómeno propositivo dentro del contexto, como base para la investigación.

Al indagar en el análisis de este suceso, se pudo observar que el propósito que más incidencia tenía, dentro del logro de objetivos, se relacionaba con la transformación del pensamiento de los alumnos/as, de modo que este trascendiera a la asignatura y el ámbito profesional a futuro. Para ello, se analiza la actividad principal y las acciones que se ejercen en la obtención de dicha meta.

Siguiendo los trabajos de Leontiev, Kuutti (2001) se plantea que la actividad tendría por finalidad objetivar las necesidades traduciéndola en un objeto, el cual -como ya se ha señaladooperaría como motivo de la actividad. Este autor sostiene que en la operacionalización de dicho proceso, las actividades se traducirían en:

a. La Actividad en tanto que unidad; como categoría global incluiría al conjunto del proceso recursivo, que iría desde el reconocimiento de la necesidad hasta su desemboque objetivado en un motivo, que incluye no sólo la actividad individual sino también la actividad 
colectiva, definida como algo hecho por una comunidad, con un motivo que necesitó ser reconocido conscientemente.

b. Acciones; las que estarían subordinadas al logro de metas específicas, con atributos conscientemente definidos, para cuyo fin podría requerirse el concurso y la articulación de una variada gama de acciones, las que a su vez se traducirían en variadas operaciones.

Operaciones; realizadas por las personas sobre distintos mediadores de la actividad, determinadas por las condiciones objetivas de realización, mayormente prescritas por las condiciones instrumentales y contextuales, al interior de las cuales esta actividad se desarrollaría.

En el caso que nos ocupa, nos referimos a la actividad en tanto que unidad (a.) la enseñanza de Ética en la Universidad de Santiago, dentro de la FACTEM. Las acciones (b.) subordinadas al logro de metas específicas, que en esta investigación se detectaron tres fases: I) Programar, II) Impartir, III) Evaluar. Las operaciones (c.) que surgen dentro de esta dinámica y que serán abordadas más adelante para detectar competencias del estudio.

A esto se suman los aportes de la psicología ergonómica rusa, a través de los trabajos de Leontiev (1983) y Ochanin (1981), la cual tempranamente se preocupó del rol de la actividad desarrollada por las personas en situación de trabajo sobre el desarrollo de sus representaciones operatorias, para los efectos de implementar estrategias de capacitación laboral y gestión de la carga de trabajo mental resentida por los operadores humanos. Dicha funcionalización de las representaciones orientadas a la acción (Weill - Fassina, Rbardel y Dubois, 1993), involucraría una deformación atingente de la realidad y de los objetos hacia los que se dirige la actividad. Según Ochanin (1981), en este proceso constructivo de representaciones operatorias se distinguen 3 fases:

- Una primera fase que identificaría a las personas carentes de experiencia, estableciendo dificultades para las relaciones operatorias significativas entre los elementos de la situación, desarrollando modalidades de ensayo y error como mecanismo de acción.

- Una segunda fase, propia del desarrollo un conocimiento funcional básico, en la que la actividad estaría marcada por las relaciones operatorias evidentes que ofrece la situación y que favorecen el desarrollo de representaciones mentales operatorias adaptativas, marcadas por las posibilidades objetivas y prescritas de la situación.

- Una tercera fase de aproximación propositiva, más propia de aquellas personas experimentadas, en donde se produciría un vuelco en la relación que las personas obtendrían con los escenarios de acción, pasando de una relación adaptativa a una relación transformadora, en la cual son las personas las que organizan los recursos de la situación en función de sus propios propósitos.

Desde una visión constructivista, se concibe entonces que la función del conocimiento no es representar o proporcionar una descripción del mundo exterior, sino que organizar el mundo experiencial del sujeto de forma que asegure suficientemente la supervivencia. (Alfaro, 2000). Es así que a medida que los recursos se organizan, aparecen las acciones y sus consecuentes operaciones para su consecución, observándose en este último punto las competencias, para esta investigación. Por tanto, en base a la literatura, no deben identificarse antes de la formación para el trabajo, pues ésta, dada su necesaria orientación a la acción, conlleva a la modificación de las competencias iniciales e incluso a la generación de competencias nuevas en los trabajadores (Cariola \& Quiroz, 1998).

Bajo el mismo paradigma, la actividad es entendida como aquello que los operadores humanos realizarían efectivamente para llevar a cabo una tarea, lo cual es distinto a lograr un objetivo determinado por la organización (Leplat, 2001).

El concepto de actividad, elaborado a la sazón de la corriente inaugurada por Lev Vygotski durante el primer tercio del siglo $X X$, ofrece una base comprensiva fértil y pragmática para la psicología laboral y organizacional contemporánea. En particular, permite situar e integrar la actividad de las personas en y con su contexto histórico, social y cultural. Esta perspectiva plantea la idea de que la actividad humana constituye un proceso continuo que une necesidades, motivos, pensamiento y comportamiento, articulados por medio de las herramientas simbólicas que la especie humana ha ido desarrollando a lo largo de su Historia. El ordenador -entendido como herramienta en el sentido dado por Vygotski (1934)- introduce una forma de interacción con las informaciones, el conocimiento y con otras personas totalmente nuevo y diferente a otros medios utilizados hasta el momento. Además de los artefactos, Vygotski (1934) enfatiza en la importancia del aprendizaje dentro del grupo a partir del concepto de Zona de Desarrollo Próximo, que es la distancia entre el nivel de desarrollo actual determinado por la solución independiente de un problema y el nivel de desarrollo potencial, determinado mediante la solución de un problema bajo la guía de un adulto o la colaboración entre iguales. Es más, existe un enfoque desarrollado a partir de la teoría de la actividad (Engeström, 1999), que considera la ZDP desde un punto de vista colectivista, definiéndola como la distancia entre las acciones cotidianas de las personas y una nueva forma de actividad social que puede ser generada colectivamente como una solución del doble vínculo potencialmente incorporado en dichas acciones.

Basándose en la teoría Histórico Cultural de Vygotski, se le adjudica a Leontiev ser precursor de la Teoría de Actividad Humana (Paez, 1996). Este enfoque consiste en concebir la realidad como un conjunto de realidades mediadas socialmente y se caracteriza por la presencia de una noción de conocimiento "proactivo" (Fried \& Fuks, 1994, citado en Alfaro, 2000), que lo entiende como una construcción activa del individuo o la colectividad en el encuentro («choque») del organismo con el ambiente, no por medio de los sentidos o la comunicación (Alfaro, 2000).

Se indica que la actividad sería "un sistema coherente de procesos mentales internos, comportamientos externos y procesos motivacionales combinados y dirigido al logro de metas conscientes" (Bedny \& Meister, 1997, como se citó en Díaz, 2008). Así, las necesidades y motivos energizarían la actividad que desarrollan las personas, estimulando la organización y despliegue de los procesos mentales. No obstante y a pesar del correlato entre necesidades y motivos, no responderían en forma directa a esta activación, ya que una misma meta podría servir a diversos motivos, porque la actualización de 
los procesos mentales se encontraría socialmente mediada. De acuerdo a esto, Weill-Fassina, Rebardel y Dubois (1993) nos señalan que la funcionalización de las representaciones orientadas a la acción involucraría una deformación atingente de la realidad y de los objetos hacia los que se dirigiría la actividad. De este modo, la construcción de representaciones operatorias funcionalmente adecuadas sería concebida como el resultado de un proceso constructivo.

El estatus de las diversas manifestaciones de la actividad podría así ser definido en función de su rol en el proceso tendiente al logro de metas, evitando así la reificación de las expresiones del comportamiento y de los procesos mentales (por ejemplo en la definición del estatus de la exploración visual, de los movimientos de las manos o de cálculos mentales), los que podrían ser analizados comprensivamente según su rol funcional al interior de la actividad finalizada de los individuos (actividad dirigida a objetos) (Diaz,2008). Entonces, la distinción entre una actividad y otra respondería en definitiva a las diferencias de los motivos hacia los que se orienta (Kuutti, 2001). Cabe recalcar, como ya se ha mencionado, que desde la perspectiva de la TA los procesos descritos poseerían una marcada impronta social (lejos de constituir un fenómeno individual, aislado y comprensible en sí mismo), pues estos se inscribirían en una red cooperativa de acciones, conformada por cadenas y redes de acciones relacionadas unas con otras en torno a los objetos de la acción.

La actividad humana sería precisamente lo que configura un contexto, siendo éste algo más que el escenario físico al interior del cual se encuentran las personas, o la combinación de personas y roles que participan de una situación (Erickson \& Shultz, 1997). Estos contextos tendrían un carácter eminentemente social e interaccional, resultando tanto de las definiciones compartidas y ratificadas mutuamente entre los participantes de una situación dada, como de las acciones sociales que estos actualizan en referencia a tales definiciones. Desde esta perspectiva, opera como un factor constructivo y contingente de la actividad (Díaz, 2008).

Finalmente, existen diversas dimensiones del contexto para una actividad dada y, por extensión de ésta, una variedad de posibilidades para el juego constructivo y articulador, donde las personas ponen en expansión sus competencias y generan el desarrollo de su experiencia. Entre las dimensiones o características de la actividad, se mencionan algunas tales como (Leplat, 2004):

- Propositivo: Orientado a satisfacer necesidades, por el medio del logro de objetivos y metas.

- Contextual: Inscrito en un escenario de acción objetivo, subjetivo y procesual.

- Contingente: Por acoplamiento mutuo entre las características de las personas con las del escenario de acción.

- Constructivo: Articulando creativamente las dimensiones personales, cognitivas, socioculturales, funcionales y contextuales involucradas en el proceso de la actividad.

- Multinivel: Pudiendo expresarse en variados y simultáneos planos de acción.

- Plástica: Re-articulando y expandiendo los soportes cognitivos que sustentan la actividad, y redefiniendo la jerarquía de los planos que concurren en ésta.

- Evaluativa: Centrada en el reconocimiento del estándar esperado.
- Proyectiva: Centrada en la evolución de los procesos en curso (Díaz, 2008).

\section{Método}

Para comprender la actividad que los docentes ejercen al enseñar la asignatura de Ética, se hace necesario obtener información que permita vislumbrar el contexto y sus redes de acción. Basándonos en ello se hace un diagnóstico de sus operaciones, principalmente a partir de las fallas y carencias que los mismos docentes expresan, visualizando alternativas para el logro de las metas y, por ende, proponiendo competencias para ello.

- Reconocer, a partir del contexto inserto dentro la asignatura de Ética, contenidos y modalidades críticas de operación que impidan perfeccionar su desarrollo.

- Describir, desde este reconocimiento, competencias requeridas para el ejercicio de la enseñanza en Ética, en la FACTEC de la USACH.

Desde una estrategia cualitativa y utilizando el concepto de la actividad docente en Ética, se hizo relevante la elaboración de una metodología de corte interpretativo-participante. La metodología cualitativa refiere, a procedimientos que posibilitan una construcción del conocimiento que ocurre sobre la base de conceptos, siendo éstos los que permiten la reducción de la complejidad, mediante el establecimiento de relaciones entre ellos para generar coherencia interna del producto científico, (Krause, 1995).Finalmente, el diseño de esta investigación corresponde a uno de tipo exploratorio, buscando indagar en un área poco explorada en estudio (Sampieri, 2014).

Cabe señalar que la investigación fue de corte etnográfico, pues los acontecimientos se conciben tal y como los expresan sus informantes, mediante la exploración de su pensamiento en su accionar (Krause, 1995). El análisis del discurso es interpretativo, pues la realidad queda asignada al significado que los actores sociales, le otorgaron (Guba, 1990, citado en Krause, 1995).

\section{Participantes}

Este trabajo contó con la participación de 10 entrevistados, cuya recolección de datos fue a partir de 10 entrevistas en profundidad y 2 grupos focales (Valles 2002). Estos últimos sirvieron como complemento a la información entregada en las entrevistas. Para buscar a los participantes, se partió por la premisa de muestra intencionada (Argibay, 2009), dada la necesidad que los entrevistados tuviesen experiencia de al menos 3 años ejerciendo Ética en pregrado. Para dicho objeto se entrevistaron a 10 Profesores de Ética de pre y post grado docentes de la asignatura en Universidad de Santiago, entre los cuales cuentan 8 psicólogos, 1 ingeniero comercial y 1 administrador público.

El tamaño de la muestra fue definido por saturación, entendida como el punto ante el cual se ha escuchado ya una cierta diversidad de ideas y con cada entrevista u observación adicional no aparecen nuevos elementos (Martínez, 2012). 


\section{Procedimiento}

La elección de los participantes se vio condicionada por la disposición e interés hacia la asignatura de Ética, que estuvieran de acuerdo en destinar parte de su tiempo a conceder una hora de entrevista y a participar en un grupo focal de 1 hora. El segundo grupo focal se concibió mediante la participación en el Seminario denominado "Motivaciones e Impresiones Generales respecto a la Formación en Ética", realizado en la Escuela de Psicología de la USACH el año 2011. Se ocupó la Teoría de la Actividad para el desarrollo de la entrevista, estableciendo significados importantes y herramientas para el logro de objetivos como elementos para su construcción.

Se aclara a los participantes que el estudio sólo tiene énfasis en incrementar el conocimiento científico y profesional, para propiciar el bienestar de quienes realizan la asignatura, tomando en cuenta que los resultados del estudio pueden ser de su conocimiento y uso, protegiendo la identidad de quienes colaboraron. No se usó consentimiento informado por escrito.

\section{Análisis}

Se utilizó el análisis de Contenido, cuyo procedimiento es axial, ya que en una primera instancia se trabaja con categorías predefinidas desde la Teoría de la Actividad, considerando sólo su dimensión propositiva (Valles, 2000, citado en Cáceres, 2003). Los datos obtenidos fueron analizados conforme a la definición de la dimensión propositiva, que contempla cuales son las necesidades a satisfacer por quien desarrolla la actividad, para el logro de objetivos. Sumado a ello, los planteamientos de Leontiev (1983) permiten ordenar la actividad como unidad y derivarla en acciones que son llevadas a cabo conforme a operaciones (Kuutti, 2001). Por último, los aportes de la ergonomía francófona a partir de Ochanine (1983), permiten jerarquizar las operaciones de acuerdo al grado de experticia y reconocimiento de la realidad de la actividad de los entrevistados.

\section{Resultados}

En función de la corriente teórica desarrollada por Vygotski (1934), en conjunto con su colaborador Leontiev (1983), se trabaja esta investigación en función de metas y objeto a transformar. A consecuencia de ello, se obtiene que la meta en cuestión es ejercitar y preparar el pensamiento del alumno (objeto), transformándolo dentro de su plano profesional y personal, trascendiendo de este modo la clase y la asignatura.

Algunas menciones en este sentido:

"Es entregar un ladrillo de pensamiento, sino estás en clase no importa porque está internet, la clase de ética no está en internet es la posibilidad de tener espacio, no tengo otro profe con quien conversar lo las drogas..."

"A mí me interesa un trabajo que se oriente a la reflexión... eee... coordinando el contenido de aporte teórico que facilite esa reflexión que le den un soporte."

"La asignatura puede intervenir en el contexto futuro al entregarle a los alumnos pautas en proyectos a desarrollar de forma didáctica... y de evaluación."

Como dato aleatorio, cabe mencionar que dentro de los mismos docentes no existe claridad ni argumento sólido que sustente qué hace que un profesor pueda ejercer la asignatura de Ética:

"... si no tendríamos a un médico sacando dientes o un psicólogo haciendo tortas, que se puede hacer, pero el tema de ética, si no sabe del contexto, no sé si lo podría hacer en esa carrera en particular, no sé."

"Yo partí haciendo ética porque los chiquillos me dijeron, Ud. nos va hacer ética el próximo semestre..."

Siguiendo con los argumentos de (Kuutti, 2001), se realiza un ordenamiento de las distintas expresiones halladas en torno a categorías, cuyo grado de pertinencia está vinculado con el proceso de transformación del objeto. Finalmente, se realiza una operacionalización de dicho proceso, en donde las actividades se traducirían en acciones, y estas a su vez en variadas operaciones.

Para dar una mayor comprensión a este proceso, se relaciona a la actividad en tanto que unidad, con aquella categoría global reconocida conscientemente mediante la enseñanza de la asignatura y que busca cambios significativos a nivel del pensamiento en el alumno/a FACTEC. Segundo, las acciones que se subordinan a dicho proceso se identificarían como: I) Programar, II) Impartir y III) Evaluar la asignatura de Ética, todas las cuales se pueden constatar en las siguientes declaraciones:

"Al identificar el programa, sus objetivos, conceptos, dinámicas, metodologías de evaluación, etc., reviso mi propio material y trato a su vez de ver qué impacto podrían tener dichos objetivos y programa en general en el alumno."

"Yo hago muy bien las clases; así que no tengo problemas para conversar de cualquier cosa, menos de hacer las clases." "Una es esa y la otra es la calificación del 1 al 7 que es fácil; el problema es cómo se hacen los instrumentos para constatar si leyeron o no y si entendieron."

Las cuales se retroalimentan e interrelacionan entre sí, en un proceso continuo para el logro del objetivo del profesor.

En tercera instancia, cabe mencionar que las operaciones realizadas por los docentes, sobre los distintos mediadores de la actividad, es el centro de esta investigación; las que a su vez van prescritas por las condiciones contextuales donde se desarrolla la actividad (Kuutti, 2001).

En función de esto último y una vez que son detectadas, se señalan otras tres fases que van desde la falta de experiencia, marcada por la dificultad para establecer relaciones operatorias significativas entre los elementos de la situación, hasta la experticia con una completa apropiación propositiva del objeto y su transformación (Ochanine, 1981).

Para ello, el análisis de las acciones dentro de la actividad como unidad permite responder a inquietudes presentes dentro de los objetivos planteados en las operaciones. 


\section{Primera Acción - Programar la Asignatura.}

A. A la luz de los resultados, el contexto en que se enmarca y desarrolla la actividad hace plantearse al docente el dilema de diseñar o adaptar un programa, que satisfaga las inquietudes del tipo de alumnado al que se imparte la asignatura, generando un impacto en éste, tanto en su calidad de estudiante como de futuro profesional. Para ello, el docente debe ir identificando situaciones culturales que tengan relevancia dentro de la sociedad y diseñar dilemas éticos que los alumnos/as puedan ir relacionando con la bibliografía sobre ética.

Lo anterior debe prepararse de tal modo que los temas más sensibles se dejen para el final, para de esta forma no irrumpir con problemáticas complejas para el alumno y que éste se sienta con más confianza al opinar. De esta forma, para alcanzar metas tales como la estimulación del pensamiento crítico, el docente se ve motivado a incluir todas estas variables en su accionar, desarrollando la programación conforme a lo que intuitivamente estime conveniente. Dado esto, es posible distinguir las siguientes operaciones y/o competencias que los docentes despliegan a partir de la acción: Conocer una amplia bibliografía, con autores tales como psicólogos o filósofos en el ámbito de la ética; identificar el tipo de alumnado con el que debe trabajar, a partir de su sensibilidad y experticia; adecuar los contenidos, partiendo de problemáticas de menos a más complejas o sensibles; generar debates y diálogos, en los cuales el alumno ejercite su capacidad de ponerse bajo distintos puntos de vista; identificar y desarrollar temas de interés para su curso.

Las siguientes citas, extraídas de entrevistas y grupos focales, permiten reconocer el contexto inserto y sus respectivas modalidades operacionales, a partir de las cuales se pueden detectar sus competencias, las que a su vez señalan el nivel de desarrollo actual dentro de su Zona de Desarrollo Próximo, Vygotski (1934).

"Eee... bueno me pregunto en primer lugar los objetivos que quiero lograr, me planteo la caracterización de grupos de trabajo para saber la metodología apropiada..."

"Hago muchas actividades que puedan trabajar en forma grupal y voy observando en forma grupal... los voy apoyando..." "En los aprendizajes hay que rescatar las vivencias de los alumnos, las vas leyendo. Hay muchos elementos que son interesantes, sabrosos, respecto a las realidades del alumno."

"Una de mis fortalezas es que soy bien empático, entonces yo lograba entender lo que querían expresar los chiquillos... sino fuera empático no lo lograría y probablemente generaría un problema mayor."

"Pequeña exposición no es cierto donde vayan soltándose... que es una complejidad... generándoles confianza, porque lo primero que te encuentras es que no hablan no opinan. Las primeras semanas..."

B. Si bien las operaciones planteadas anteriormente presentan dominio por parte de los docentes, debido a la experticia y la facilidad para establecer relaciones operatorias significativas de los elementos mencionados en la situación, existen modalidades críticas que evidencian falencias de domino en las representaciones operacionales, conforme a la actividad de enseñar en la asignatura de Ética, Ochanine (1981). Tal es el caso del diseño de un programa, que contemple material educativo extensivo acorde a cada situación y un marco teórico que ordene basalmente la asignatura para encauzarla. Por ejemplo, desde una visión económica, política, psicológica, sociológica, etc., conforme a cada carrera.

En este sentido, no se observa el uso de metodologías propicias y sólidas para la entrega de unidades que se sustenten en curvas de aprendizaje, procedimientos de Ajzen (teoría de la acción razonada), por citar algunos ejemplos. Adicionalmente, tampoco se detecta el diseño de clases conforme a una metodología adecuada para alumnos/as que están en una etapa inicial de aprendizaje, y que poseen dificultades para generar representaciones significativas. Si bien los profesores tienen dominio de material bibliográfico, no buscan relacionar este material conforme a las necesidades propias de la madurez de los estudiantes, sensibilidades, curvas de aprendizaje, entre otras características. Más bien aparecen dentro de un discurso, como un conocimiento funcional básico marcado por relaciones operatorias evidentes de la situación. Estos temas son mencionados en mayor o menor medida por los docentes, los cuales no especifican modalidades de abordaje claramente definidas.

\section{Segunda Acción - Impartir la Asignatura}

A. Al igual que en el proceso anterior, basándonos en la dimensión propositiva de la actividad docente, se pueden distinguir acciones mediadas por varias metas a lograr en el alumno/a. Una de ellas es no imponer un tipo de pensamiento, sino el desarrollo de uno propio, y a través de ello la generación de diversos conocimientos y herramientas que, una vez en la vida profesional, entreguen un universo amplio para actuar éticamente en base al grado de transformación moral.

Así, una clase que sólo contenga doctrinas, códigos, leyes y/o teoría no permitirá al alumno hacer la distinción entre libertad, sumisión y/o responsabilidad; menos aún si sólo se tiene un semestre para impartir la asignatura. Otras metas tienen que ver con dominar técnicas que permitan el desarrollo de la confianza en clase, para generar condiciones de empoderamiento a la hora de afrontar posturas y posibilidades, seguridad para argumentar, capacidad para orientarse intuitivamente en temas éticos, poseer sensibilidad respecto a éstos, saber actuar y desenvolverse en situaciones complejas, tener un criterio formado, ser entretenido, ser honesto, empático, conocer amplia bibliografía, tener manejo de grupos, entre otras similares que aluden a virtudes personales.

Las siguientes citas extraídas de entrevistas y grupos focales, permiten reconocer el contexto inserto y sus respectivas modalidades operacionales a partir de las cuales se pueden detectar sus competencias, las cuales a su vez señalan el nivel de desarrollo actual que, dentro de este trabajo, busca responder a su Zona de desarrollo potencial (1934).

"... si los jóvenes no están formados, por lo tanto hay que formarlos!!... No les voy dejar que se desarrollen como son, voy a imponerles mi punto de vista, voy a decirles lo que tienen que hacer, los voy a moldear." (fue dicho en tono de sarcasmo)

"... o enjuiciador, está ese riesgo de decir esto es inaceptable, o esto es no porque no, prejuicios..." "...mmm... de ponerse 
en el lugar del otro, que alternativas se tenían, si había o no posibilidad de decidir cuáles eran los efectos de la decisión."

"El hecho de que ellos se den cuenta que ética no es una asignatura, yo los invito a hacer otra cosa como parte de su desarrollo personal, profesional y laboral por eso me interesa mucho que ellos analicen también su perfil de egreso y veamos el tema de actitud y valores."

De acuerdo al análisis hecho conforme a la actividad desarrollada por los docentes, las competencias que se observan en una primera instancia se desglosan en: Conocimiento de técnicas variadas que rompan el hielo del alumno; generación de empatía con los alumnos/as; actualización de la práctica docente en técnicas de aprendizaje atingentes; conocimiento de aquellas situaciones de transgresión que más impactan al alumno; creación de herramientas de aprendizaje que hagan al alumno relacionar su mundo externo con el interno; sensibilidad para darse cuenta cuando una dinámica hace daño; capacidad para relacionar conceptos con temas éticos de interés para el alumnado; entrega de contenidos abordando diversas visiones culturales; respaldo de opiniones en función de argumentos sólidos a partir de autores procedentes de la psicología y la filosofía.

B. No obstante, al remitirnos a las modalidades operacionales críticas dentro de la dinámica de este contexto, podemos darnos cuenta que no existe experticia dentro de la relaciones operacionales mentales que tengan que ver con lo expuesto en la programación. Es decir, todo aquello que no se vislumbra en la programación, tampoco se ve reflejado en la impartición.

Aparecen aspectos que son evidentes y básicos en cuanto a la apropiación de la realidad y transformación del objeto, tales como: Utilización de técnicas que rompan el hielo con los alumnos/as; búsqueda de empatía con el curso; utilización de técnicas y dinámicas que no vulneren ni sean maleficentes (como juegos de rol que sean dañinos para el alumno por haberlos vivido en persona); libertad de creencias y conocimientos conceptuales, en cuanto a lo justo o injusto.

Dentro del domino de las relaciones operatorias, estos elementos tienen que ver más con representaciones mentales declarativas, ya que si bien lo saben, no es en un nivel que domine la transformación del objeto, tal como se señala en una tercera fase de apropiación operacional de ésta, Ochanine (1981). Lo anterior se debe a que no hay evidencias contundentes de un trabajo concienzudo, planificado e integrador, conforme al diseño del programa y la impartición de la asignatura, como pilar de las operaciones mencionadas por los docentes.

Finalmente y siguiendo con el análisis que se mencionó anteriormente, no hay una relación operatoria definida entre lo que se hace en clase y lo que hay en el programa, evidenciado en la variedad de discursos presentes.

\section{Tercera Acción - Evaluar la Asignatura}

A. Finalmente, cuando llega este episodio que contempla la actividad, la complejidad se hace mayor. Inmediatamente aparece la disonancia entre calificación vs. evaluación, es decir, por una parte puede ser que los alumnos/as tengan excelentes calificaciones, pero se aprendan todo de memoria.
Si observamos esta dinámica, podemos darnos cuenta de que las habilidades que presentan los docentes para lograr evaluar, tienen que ver con diseñar pruebas cuyo objetivo de aprendizaje esté basado en que el alumno sea capaz de analizar, sintetizar e integrar una solución a problemas éticos, en las que la corrección de los argumentos sea sólida y tenga que ver con el pensamiento crítico. Adicionalmente, que estas pruebas posean un contenido que tenga sentido para la vida personal y profesional, evitando generalizaciones y clichés. Las siguientes citas extraídas de entrevistas y grupos focales, permiten reconocer el contexto inserto y sus respectivas modalidades operacionales a partir de las cuales se pueden detectar sus competencias. Las cuales a su vez señalan el nivel de desarrollo actual, dentro de su zona de desarrollo potencial, Vygotski (1934).

"Mi evaluación es hacia mí mismo, si lo estoy haciendo bien o no, con ganas o no, qué tanto están motivados por ir o por participar o por lo que te dicen aprendí, gracias profe."

"Yo tengo 2 puntos, una es la evaluación y la otra es la calificación que no hay problema del 1 al 7, es fácil. El problema es cómo se hacen los instrumentos de constatar, a través de un cierto tipo de preguntas o situaciones, primero, si leyeron el texto; segundo, si entendieron algún concepto, y tercero, si es que pueden escoger aquella que es la correcta."

"¡Al evaluar! Trato de ver cómo evaluar... pero no puedo calificar a un chico que está más atrás en su desarrollo, todo lo contrario, debo estimular ese desarrollo y se me produce un problema..."

Desde esta perspectiva y tomando como referente adicional la ergonomía cognitiva, aquello que aparece como carencia en la programación también repercute en la acción de evaluar, ya que no existe discurso alguno que la mencione; es decir, no se hace una relación coherente entre la entrega de contenidos y el tipo de evaluación conforme al grado de representación operatoria. Se observa dificultad para las relaciones operatorias significativas, presentándose baja articulación y reconocimiento de atributos relevantes dentro las operaciones para lograr evaluar. Aquí la detección de competencias se condensa en una falencia frente al contexto, lo que se hará presente en el siguiente apartado.

\section{Discusión}

Desde la perspectiva de Vygotski (1934), se enfatiza la importancia del aprendizaje dentro del grupo, a partir del concepto de Zona de Desarrollo Próximo (ZDP). Esta constituye la distancia entre el nivel de desarrollo actual, determinado por las solución independiente de un problema, y el nivel de desarrollo potencial, determinado mediante la solución de un problema bajo la guía de un adulto o la colaboración entre iguales (Berk, 2006). Para indagar aún más en esto, también se menciona la ZDP como la distancia entre las acciones cotidianas de las personas y una nueva forma de actividad social, que puede ser generada colectivamente como una solución del doble vínculo potencialmente incorporado en dichas acciones (Engeström, 1999). Bajo el alero de esta propuesta, es que se detecta que los profesores enmarcan su actividad dentro de un contexto en el cual van modelando su accionar. A diferencia de lo que ocurre con Vygotski, las actividades docentes analizadas no cuentan con un patrón modelo al cual seguir, 
sino que se van dando por medio de acciones cotidianas y que se van cristalizando a medida que van siendo adecuadas para obtener un propósito, por medio de la rutina, que con el tiempo se torna estructurante del contexto (Díaz, 2008).

Esto podría explicarse debido a la ausencia de criterios establecidos conforme al tipo de profesional que debiese impartir la asignatura, el cual puede ser psicólogo, médico, ingeniero, etc. Por otro lado, tampoco existe un manual específico para dictar la cátedra en Ética de cada una de las diversas carreras y especialidades. Al contrario, cada profesional, cuya procedencia académica corresponde a distintas áreas, opera según sus conocimientos, experiencia y motivaciones personales. Este hecho, en cierta medida, resta uniformidad a la programación, impartición, evaluaciones y al mismo tiempo al sello institucional.

En relación con lo expuesto y debido precisamente a la carencia de uniformidad, para comenzar el análisis se detectó que, independiente de la formación del docente, el objetivo era el mismo. No obstante, esto fue insuficiente para identificar, desde el contexto en el cual se enmarca la actividad, qué competencias debe tener un docente para lograr su propósito. Incluso hasta el momento sólo se puede hablar de los profesores, ya que no se ha hablado de las necesidades de los propios alumnos/as, o de los perfiles de egreso que plantean los organismos de la educación superior.

Para profundizar en el conocimiento de la dinámica de este contexto y siguiendo los planteamientos de Leontiev (1983), se realizaron categorías que permitieron dar un ordenamiento a la relación entre profesor y alumno dentro del contexto, partiendo por la actividad en tanto unidad (Kuutti, 2001), como la transformación del pensamiento del alumno por parte del profesor, a través de la enseñanza en clase. Posteriormente a nivel de las acciones, se detectaron 3 acciones importantes, que parten por la programación, siguen con la impartición y por último evaluación, todas ellas unidas en un continuo relacional. Una vez consideradas estas actividades, se sustentan las operaciones dentro de ellas, las cuales permitieron observar aquellas modalidades críticas de acción o aquellas dificultades que presentaban los profesores para establecer relaciones operatorias significativas, las cuales fueron sostenidas desde sus propias necesidades dentro del discurso. También se utilizaron teorías concernientes al desarrollo moral, curvas de aprendizaje, estilos de aprendizaje, normatividad, entre otras; todas las cuales aparecían en el relato de los entrevistados.

Se puede reparar en que los profesores sí tienen experticia en varios ámbitos de la actividad, la cual permite hacer una propuesta de competencias en base a las operaciones realizadas para el logro de la meta. No obstante, si es que se desea transformar el pensamiento crítico del alumno, queda en evidencia que estas competencias no son suficientes para lograrlo, ya que no se establece una estructura o marco teórico conforme al programa, que logre la modificación del pensamiento crítico mediante una planificación anticipada del curso, ni que esta programación tenga relación con la impartición y menos con el apartado de la evaluación.

A partir de los lineamientos propuestos para dar forma y estructuración al contexto formativo, se evidencia que lo único observable dentro de las representaciones operacionales de los docentes tiene que ver con los distintos aportes personales que cada profesor pueda facilitar. Por ejemplo, la programación se lleva adelante a modo discrecional, acorde a las diversas profesiones y experiencias personales, ante lo cual se detectan debilidades en el orden y la metodología utilizados para impartir los contenidos y sus fundamentos teóricos. Desde este contexto propositivo (Díaz, 2008), observamos a un docente que pone gran parte de su energía y tiempo en ir flexibilizando, adaptando y adecuándose a cada necesidad específica. No obstante y en base a las modalidades críticas halladas en el desarrollo de esta dinámica, esto toma la forma de un ejercicio de ensayo- error sin fin, restando fuerza a la transformación del objeto, manteniendo amplia la brecha entre lo que hacen y su desarrollo potencial.

Además, se cuestiona la capacidad de transformar el pensamiento crítico del alumno, o sea si es efectiva o se deforma, ya que no se puede determinar algún grado de medición al respecto. Esto se debe a la poca pertinencia entre las tres acciones, porque no se sabe exactamente lo que se mide ni como se mide, generando aún más incertidumbre si estos aprendizajes trascienden el escenario de la sala de clases, el tiempo y el área personal.

Si bien existen representaciones operacionales que utilizan los docentes para el logro de sus metas, se encuentran deficiencias en cuanto a: (1) Un marco teórico que ordene basalmente a la asignatura para encausarla (por ejemplo, desde una visión económica, política, psicológica, sociológica, conforma a cada carrera, etc.). (2) El legítimo dominio bibliográfico que debiesen manejar, conforme al diseño de programas adecuados, acordes a las necesidades propias de su madurez y los marcos teóricos antes mencionados. (3) El uso de metodologías propicias y sólidas para la entrega de unidades que se sustenten, por ejemplo, en curvas y estilos de aprendizaje, procedimientos de Ajzen (teoría de la acción razonada), etc., y que estén descritos en los programas. (4) Coherencia entre lo programado, lo impartido y lo evaluado. (5) La evaluación conforme al nivel de representación operacional del alumno, acorde al avance de unidades dentro del programa. Un mal diseño de programa, abriría la puerta a la de-formación del alumnado, más que a su formación, pese a las buenas intenciones del docente.

De igual forma, falta información respecto al conocimiento que se tiene sobre el comportamiento ético de los egresados en el mundo laboral, así como de sus falencias en este ámbito. Por otra parte, es relevante conocer las carencias teóricas en los programas, insuficiencia de metodologías de aprendizaje, así como niveles significativos de contenidos curriculares. Finalmente, a lo anterior se suma que tampoco están contempladas las necesidades de los alumnos/as, ni las necesidades de la malla curricular que podrían responderse, con investigaciones de este tipo.

En este marco, se sugiere fortalecer la coherencia entre las evaluaciones y cada etapa de aprendizaje. De esta manera sería más fácil medir las transformaciones en el pensamiento crítico y el actuar de un estudiante, acercándose más a la meta que motiva a los docentes, ya planteada anteriormente. Como respuesta a los objetivos, se proponen nuevas competencias a desarrollar que sean complementadas por aquellas que ya presentan los docentes, tales como: 
1. Comprender el tipo de marco teórico que debe definirse para el tipo de alumno/a, conforme a su carrera y desarrollo moral.

2. Manejar bibliografía, para elaborar un programa en concordancia a lo anterior.

3. Desarrollar diversos métodos y técnicas de entrega de contenidos, conforme al nivel de aprendizaje, desarrollo y nivel motivacional del alumnado.

4. Manejar dilemas éticos dentro del desarrollo evolutivo de la moral, en situaciones contingentes al área personal, social y laboral.

5. Diseñar la entrega de contenidos a nivel conceptual, metodológico (por ejemplo, estructurar planes de acción conforme a teoría, códigos, leyes, etc.) y contextual (por ejemplo, en base a videos, entregar contenidos que tengan relación con el accionar, etc.).

6. Diseñar tipos de evaluaciones coherentes a esta entrega de contenidos y etapa de aprendizaje en que se encuentra el curso.

7. Conocer diversas técnicas para el desarrollo de estas metodologías, tales como uso de bibliografía, informes, exposiciones, películas, paneles-debates, role playing, estudios de caso, entrevistas en empresas, investigaciones, videos formateados con errores éticos, entre otras.

En paralelo, se advierte la necesidad de investigar dentro de la actividad y el contexto académico, para levantar propuestas desde los estudiantes y desde las instituciones de educación superior, que puedan sustentar efectivamente los requerimientos reales de una malla curricular, e incluso enriquecerla y complementarla.

\section{Referencias}

Alfaro, J. (2000). Discusiones en Psicología Comunitaria. Santiago, Chile: RIL Editores.

Argibay,J. (2009). Muestra en investigación cuantitativa: The sample in quantitative research (versión electrónica). Revista Subj. procesos cogn. vol.13 no.1.

Barragán, R. \& Buzón, O. (2004). Desarrollo de Competencias específicas en la materia tecnología educativa bajo el marco del espacio europeo de educación superior. Revista Latinoamericana de Tecnología Educativa, 3(1), 101-114.

Berk, L. (2006). Desarrollo del Niño y el Adolescente (2a ed.). Madrid: Editorial Pearson.

Cáceres, P. (2003). Análisis cualitativo de contenido: Una alternativa metodológica alcanzable. (versión electrónica). Revista Psicoperspectivas, vol. II (pp. 53 - 82)

Cano, M. (2008). La evaluación por competencias en la educación superior. Profesorado. Revista de Currículum y Formación de Profesorado, 12(3), 1-16. Recuperado de https://www.ugr.es/ recfpro/rev123COL1.pdf

Cariola, L. \& Quiroz, A. (1998). Competencias generales, competencias laborales y currículum. En M. Novick \& M. A. Gallart (Coord.), Competitividad, redes productivas y competencias laborales (pp. 51-77). Montevideo: oit/cinterfor.

Díaz, C. (2008). Actividad, Contexto Organizacional y Competencias. Revista Psicología Organizacional Humana, 1(2), 53-68. Recuperado de https://es.scribd.com/ doc/53314377/Diaz-Actividad-contexto-organizacional.

Engeström, Y (1999). Activity theory and transformation In Engeström, Y, Meiettinen, R. \& Punamaki R. L. Perspectives on activity theory. Cambridge University Press.

Erikson, F. \& Schultz, J. (1997). When is a context? Some issues and methods in the analysis of social competence. En M. Cole, Y. Engestrom \& O. Vasquez (Eds.), Mind, Culture, and Activity: Seminal Papers from the Laboratoy of Comparative Cognition. New York: Cambridge University Press.

Kuutti, K. (2001). Activity theory as a potential framework for human-computer interaction research. En B. Nardi (Ed.), Context and consciousness. Cambridge: MIT Press.

Krause,M. (1995). La investigación cualitativa: Un campo de posibilidades y desafíos (versión electrónica). Revista temas de educación nº 7, año 1995, pp. 19-39.

Leóntiev, A. (1983). Teoría psicológica de la actividad. En A. Leóntiev (Ed.), Selección de Obras de Psicología (Tomo 2, pp. 94-261). Moscú: Pedagogía (en ruso). 
Leplat, J. (2001). La gestion des communications par le contexte. Revue électronique Pistes, 3(1). doi:10.4000/ pistes.3755

Leplat, J. (2004). L'analyse psychologique du travail. Revue Européenne de Psychologie Appliquée, 54, 101-108. doi:10.1016/j.erap.2003.12.006

Maritn, I. (2001). Diálogos entre razón y sentimiento. España: Ediciones Virtualibro.

Martínez, C. (2012) El muestreo en investigación cualitativa: principios básicos y algunas controversias (versión electrónica). Revista Ciênc. \& saúde coletiva, vol.17, n.3, pp.613-619.

Ochanine, D. (1981). L'image Operative. Paris: Centre d' Education Permanente.

Páez, D. (1996). La teoría sociocultural y la psicología social actual. Madrid: Colección Cultura y Conciencia.

Prieto, J. (2012). Estrategias de enseñanza-aprendizaje. Docencia universitaria basada en competencias. México: Pearson Educación.

Sampieri, R (2014). Metodología de la Investigación. México (6 Edición): Editorial Mc Graw Hill.

Valles, M. (2002). Cuadernos Metodológicos: Entrevistas cualitativas. Madrid: CIS.

Vygotski, L. (1934). Pensée et langage. Paris: La Dispute.

Weill-Fassina, A., Rabardel, P. \& Dubois, D. (1993). Représentations pour l'action. Toulouse: Octares. 